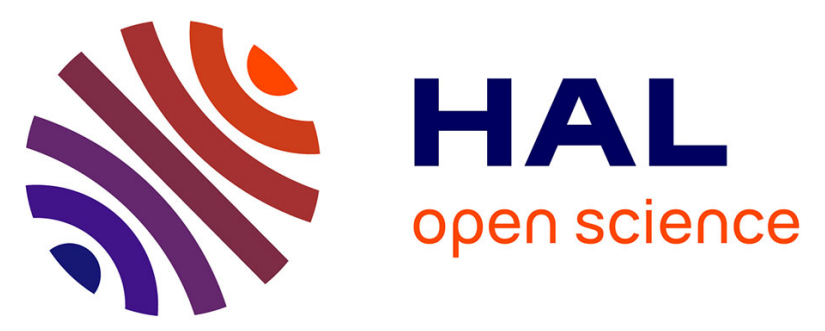

\title{
Comparaison de l'efficience entre une salle de traumatologie et une salle de chirurgie orthopédique dans un centre hospitalier universitaire
}

Thibaud Rodriguez, Aurélie Wolf-Mandroux, Jennifer Soret, Louis Dagneaux, François Canovas

\section{To cite this version:}

Thibaud Rodriguez, Aurélie Wolf-Mandroux, Jennifer Soret, Louis Dagneaux, François Canovas. Comparaison de l'efficience entre une salle de traumatologie et une salle de chirurgie orthopédique dans un centre hospitalier universitaire. Revue de Chirurgie Orthopédique et Traumatologique, 2019, 105, pp.102 - 106. 10.1016/j.rcot.2018.12.008 . hal-03486561

\section{HAL Id: hal-03486561 https://hal.science/hal-03486561}

Submitted on 20 Dec 2021

HAL is a multi-disciplinary open access archive for the deposit and dissemination of scientific research documents, whether they are published or not. The documents may come from teaching and research institutions in France or abroad, or from public or private research centers.
L'archive ouverte pluridisciplinaire HAL, est destinée au dépôt et à la diffusion de documents scientifiques de niveau recherche, publiés ou non, émanant des établissements d'enseignement et de recherche français ou étrangers, des laboratoires publics ou privés.

\section{(c) (1) $\$$}

Distributed under a Creative Commons Attribution - NonCommerciall 4.0 International 


\title{
Mémoire original
}

\section{Comparaison de l'efficience entre une salle de traumatologie et une salle de chirurgie orthopédique dans un Centre Hospitalier Universitaire.}

\author{
Compared Efficiency of Trauma versus Scheduled Orthopaedic Surgery Operating Rooms \\ in a University Hospital
}

Thibaud Rodriguez ${ }^{*}{ }^{1}$, Aurélie Wolf-Mandroux ${ }^{1}$, Jennifer Soret $^{1}$, Louis Dagneaux ${ }^{1}$, François Canovas ${ }^{1}$

1 Département de Chirurgie Orthopédique et Traumatologie, Unité de Chirurgie du membre inférieur et du rachis, Hôpital Lapeyronie, CHRU Montpellier, Montpellier, France.

\section{*Auteur correspondant :}

Thibaud Rodriguez, MD, Département de Chirurgie Orthopédique et Traumatologie, Unité de Chirurgie du membre inférieur et du rachis, Hôpital Lapeyronie, CHRU Montpellier, 351 av. Gaston Giraud, 34295 Montpellier cedex 5, France

zed34490@hotmail.com

Ne pas utiliser, pour citation, la référence française de cet article, mais celle de l'article original paru dans Orthopaedics \& Traumatology: Surgery \& Research, en utilisant le DOI ci-dessus. 


\section{Résumé :}

Introduction :

Dans une démarche d'amélioration des performances, nous avons comparé une salle de chirurgie orthopédique et de traumatologie selon les indicateurs des blocs opératoires.

\section{Hypothèse :}

L'hypothèse principale est que l'efficience en traumatologie est moindre qu'en orthopédie. L'hypothèse secondaire est qu' elle est moindre en traumatologie le week-end.

Matériel et méthodes :

Cette étude prospective a été menée sur 2016 en chirurgie orthopédique dans un centre hospitalouniversitaire. Le groupe A correspondait aux patients de chirurgie programmée, le groupe B aux patients de traumatologie en semaine, le groupe $\mathrm{C}$ aux patients opérés les week-ends. Les temps réels d'occupation des salles (TROS), les temps de vacation offert (TVO), les taux d'occupation des salles (TO), les délais « entrée en salle - incision » (T1), les délais « incision - fermeture » (T2), les délais « fermeture - entrée en SSPI » (T3), et la remise en état des salles (T4) ont été évalués.

\section{Résultats :}

Nous avons inclus 691 patients dans le groupe A, 819 patients dans le groupe B et 327 dans le groupe C. L'efficience du groupe B était moindre comparé au groupe A (TO : $86 \%$ vs $88 \%$; $\mathrm{p}=10^{-4}$ ). Toutes les étapes du TROS étaient rallongées dans le groupe B. Néanmoins chacune des étapes représentait un taux similaire dans les 2 groupes, seul T4 était augmenté dans le groupe B $(\mathrm{p}<0,05)$. En moyenne T1 représentait $31 \%$ du TROS, T2 représentait 34\%; T3 18\% et T4 17\%. L'efficience du groupe C était moindre comparée au groupe B (TO : $75 \%$ vs $86 \% ; \mathrm{p}=10^{-4}$ ). Les temps $\mathrm{T} 1$ et T2 étaient plus courts $(\mathrm{p}<0,05)$, et T4 était rallongé de presque $10 \%(\mathrm{p}<0,05)$.

Conclusion :

L'efficience en traumatologie était inférieure mais somme toute satisfaisant aux yeux des indicateurs. Chacune des étapes du TROS représentait un taux similaire excepté T4. L'arrivée du premier malade et le turn-over devraient être les principaux axes d'amélioration.

\section{Niveau de preuve : II, Etude de cohorte}

Mots clés : Efficience, Salle d'opération; Performance, Gestion péri-opératoire 


\section{INTRODUCTION}

La Mission nationale d'Expertise et d'Audit Hospitalier (MEAH) a développé il y a une dizaine d'années des indicateurs d'efficience des blocs opératoires inspirés des travaux de Strum [1]. Ils offrent un diagnostic détaillé de l'utilisation temporelle des salles d'opération, à partir duquel des mesures d'optimisation peuvent être mises en place, suivies et évaluées [2].

Dans notre pratique, l'efficience d'une salle de traumatologie paraît bien moindre qu'une salle de chirurgie programmée. Dans le cadre d'une démarche d'amélioration des performances, nous avons souhaité comparer une salle de chirurgie orthopédique programmée et d'urgence.

L'hypothèse principale est que l'efficience en salle de traumatologie est moindre qu'en orthopédie programmée. L'hypothèse secondaire est que l'efficience en salle de traumatologie est moindre le week-end.

\section{MATERIEL ET METHODES}

Il s'agissait d'une étude prospective menée sur l'année 2016 en chirurgie orthopédique et traumatologique dédiées au membre inférieur. La chirurgie orthopédique étant de plus en plus spécialisée ; l'organisation de l'orthopédie est, dans notre hôpital universitaire, divisée en deux secteurs. Un service dédié au membre supérieur et un autre au membre inférieur. Chacune des équipes disposent de salles pour la chirurgie programmée et pour la chirurgie d'urgence. Nous avons inclus les patients opérés des membres inférieurs sur la salle de traumatologie et sur une salle de chirurgie programmée avec les plages opératoires les plus grandes.

Le groupe A correspondait aux patients opérés dans une salle de chirurgie programmée. Le groupe B correspondait aux patients opérés sur la salle d'urgence en semaine. Le groupe C correspondait aux patients opérés en urgences les week-ends et jours fériés. Les plages horaires étaient similaires dans les groupes A et B (07h30 à 18h30). Au-delà, seules les salles d'urgences étaient accessibles. 
Le week-end et jours fériés, une salle d'urgence est disponible $24 \mathrm{~h} / 24$ correspondant au groupe C.

Les données horaires étaient récupérées sur la feuille d'écologie pour chaque intervention sur le logiciel Dxcare (Medasys( $)$ ). Ces données sont renseignées lors de l'acte opératoire par l'infirmière circulante. Y figurent les heures de préparation de la salle, d'entrée du patient, d'induction, d'incision, de la fermeture, de la réalisation du pansement et de la sortie de salle du patient permettant de calculer les différents indicateurs. Etaient exclus les enchaînements faits sur deux salles (dit en «ping pong ») ou les patients ayant été opérés durant la garde du fait de personnel moindre.

Ces données nous permettaient d'évaluer l'arrivée du premier malade, l'heure de la première incision et de calculer les indicateurs « socles » et les indicateurs «process circuit patient » de la MEAH comprenant (Figure1):

Le temps réel d'occupation des salles (TROS) qui correspond au temps entre l'entrée du malade et la remise en état de la salle pour le patient suivant.

Le temps de vacation offert (TVO) qui est le temps compris entre l'entrée en salle du premier patient et la sortie du dernier patient de la salle d'opération.

Le taux d'occupation des salles (TO) correspond à la somme des TROS divisés par le TVO.

Le délai « entrée en salle - incision » (T1) correspond au temps entre l'arrivée du patient en salle, l'induction, l'installation et le drapage du patient pour la chirurgie.

Le délai « incision - fermeture » (T2) correspondant au temps opératoire.

Le délai «fermeture - entrée en SSPI » (T3) correspondant au temps entre la fermeture et le pansement, la désinstallation du patient et sa sortie.

Le temps de remise en état de la salle d'intervention (T4). 
Le turn-over (T5) correspond au temps entre la remise en état de la salle et l'entrée du patient suivant.

L'analyse statistique était réalisée par le logiciel SPSS (IBMC). Nous avons réalisé un test T de Student entre le groupe A et B et entre le groupe B et C. 


\section{$\underline{\text { RESULTATS }}$}

Nous avons inclus 691 patients dans le groupe A, 819 patients dans le groupe B et 327 dans le groupe C. Les résultats sont regroupés dans le Tableau 1 :

Le taux d'occupation de salle en traumatologie (groupe B) était moindre par rapport à la salle d'orthopédie programmée (groupe A) (TO : $-2,8 \% ; \mathrm{p}=10^{-4}$ ).

Chaque temps d'occupation de la salle s'avérait être significativement augmenté en traumatologie.

Et cela commençait par l'arrivée du premier malade qui arrive 38 min après le premier malade du groupe $\mathrm{A}\left(\mathrm{p}=10^{-4}\right)$.

Si l'on rapportait chaque temps d'occupation de la salle au temps total du TROS (Figure2) :

Le délai «entrée - incision » (T1) représentait 32,2\% dans le groupe A contre 31,9\% dans le groupe $B(p=0,933)$.

Le délai «incision - fermeture » (T2) représentait 37,4 \% dans le groupe A et 36,8\% dans le groupe $B(p=0,247)$.

Le délai «fermeture - sortie » (T3) représentait 17,6\% dans le groupe A et 18,2\% dans le groupe $B(p=0,329)$.

Le temps de remise en état de la salle (T4) représentait 12,7\% dans le groupe A contre 15,1\% dans le groupe $B\left(p=10^{-4}\right)$.

Le turn-over (T5) était plus long de 13 min entre le groupe $B$ et le groupe $A\left(p=10^{-4}\right)$. 
L'efficience de la salle de traumatologie le week-end (groupe C) était également moindre par rapport à la salle en semaine (groupe $\mathrm{B})\left(\mathrm{TO}=-9,9 \% ; \mathrm{p}=10^{-4}\right)$.

Les délais «entrée - incision » (T1) et « incision - fermeture » (T2) étaient plus courts dans le groupe C. Les délais « fermeture - sortie » (T3) étaient comparables dans les deux groupes.

Le taux d'occupation de la salle restait cependant plus faible et cela s'explique par un turn-over rallongé de $31 \min \left(\mathrm{p}=10^{-4}\right)$.

L'arrivée du premier malade dans le groupe $\mathrm{C}$ se faisait presque une heure après l'arrivée du premier malade du groupe $B\left(\mathrm{p}=10^{-4}\right)$.

Le délai «entrée - incision » (T1) représentait 28,8\% dans le groupe C contre 31,9\% dans le groupe $\mathrm{B}\left(\mathrm{p}=10^{-4}\right)$.

Le délai «incision - fermeture» représentait $27,6 \%$ dans le groupe $\mathrm{C}$ contre $36,8 \%$ dans le groupe $B\left(p=10^{-4}\right)$.

Le délai «fermeture - sortie » (T3) représentait 19,2\% dans le groupe C contre 18,2\% dans le groupe $B(p=0,411)$.

Le temps de remise en état de la salle (T4) représentait 24,3\% dans le groupe C contre 15,1\% dans le groupe $B\left(p=10^{-4}\right)$.

Le turn-over (T5) était plus long de 31 min entre le groupe $C$ et le groupe $B\left(p=10^{-4}\right)$.

\section{DISCUSSION :}

L'efficience de la salle de traumatologie était moindre comparée à la salle de chirurgie programmée bien que celle-ci soit relativement satisfaisante aux yeux des différents indicateurs de performance. L'objectif classiquement avancé est un taux d'occupation $\geq 80 \%$. 
La salle de traumatologie le week-end avait une efficience moins importante par rapport à la salle de traumatologie en semaine en raison d'un turn-over rallongé (+31 min). Si l'on comparait les temps réels d'occupation de salle (TROS), chacune des étapes se trouvait rallongée en traumatologie.

L'heure d'arrivée du malade était plus tardive ; le temps d'induction, la durée opératoire, la sortie du malade et le turn-over étaient significativement plus longs. Néanmoins, chaque étape du temps réel d'occupation représentait un taux à peu près similaire dans les différents groupes pouvant être en lien avec la complexité du geste opératoire. En moyenne le T1 représentait $31 \%$ du TROS, le T2 représente 34\%; le T3 18\% et le T4 17\%.

La chirurgie traumatologique correspond à une prise en charge en urgence avec une organisation plus difficile à prévoir. Cela peut entraîner des défauts de préparation ou de coordination pouvant engendrer des pertes de temps et des tensions entre les équipes [3]. Ces manquements sont essentiellement dues à des erreurs de communication, comme concernant l'ordre des malades, le matériel utilisé ou le type d'installation [4-7]. Nous disposons d'un tableau d'urgence au bloc opératoire où les patients de traumatologie en attente de chirurgie sont notés avec leur identité, le service où le patient est hospitalisé, le diagnostic et le matériel nécessaire.

L'ordre opératoire était décidé par le chirurgien de garde la veille pour le lendemain afin de faciliter l'organisation. Il arrive cependant qu'une certaine latence persiste au moment de demander le transport du premier malade jusqu'au bloc opératoire en raison d'absence de consultation d'anesthésie, de disponibilité du matériel, etc.

\section{Arrivée du malade}

Dans notre série, l'heure d'incision en chirurgie traumatologique débutait 42 minutes plus tard que la chirurgie programmée. Malgré cette organisation, l'arrivée du premier patient reste imparfaite. Tout démarrage tardif retardera l'ensemble des procédures de la journée.

Les leviers sur lesquels il nous semble possible d'agir pour optimiser le démarrage des salles sont nombreux: 
- Identifier le «bon patient » pour démarrer le programme opératoire.

- Bien définir l'heure de démarrage des salles et l'inscrire dans la charte de bloc.

- S'assurer de la bonne coordination des différents acteurs contribuant à la réalisation de la première intervention.

- Prendre en compte certaines ressources qui peuvent être limitantes (brancardiers qui ne peuvent pas brancarder deux patients en même temps).

- Il faut sensibiliser les différents acteurs du bloc opératoire sur la nécessité de respecter les règles définies pour le démarrage des salles.

Cela permettrait ensuite d'identifier les causes de retard, qui peuvent être multifactorielles, soit pour apporter des ajustements, soit pour définir les mesures à prendre en cas de non-respect des règles.

\section{Induction}

Les patients de traumatologies sont potentiellement des patients plus fragiles car généralement plus âgés ou polytraumatisés. La réalisation de l'anesthésie peut donc être plus longue car plus difficile (Rachianesthésie, anesthésie multimodale ou patient venant de réanimation) et l'installation plus précautionneuse ou complexe (mobilisation en bloc, table orthopédique ou en décubitus ventral).

Les temps d'induction et d'installation sont devenus plus longs au fil des années en partie pour des raisons de sécurité (monitorage, index bispectral, cathéters veineux multiples), de confort du patient (anesthésie péri-nerveuse, installation) et des différents équipements (chirurgie arthroscopique ou mini invasive, appareils de radiographie) [8]. La réalisation de la Check-list pré opératoire n'influe pas sur ces étapes préliminaires. Elle permet même de résoudre des problèmes organisationnels [9] et diminue le risque de complications [10].

Le délai (T1) entre l'entrée du patient et l'incision était à peu près équivalent dans les groupes (32\%), ce qui équivaut à peu près la durée du geste opératoire T2 (35\%). Madni [11] retrouvaient 
25\% du temps d'occupation de la salle pour le T1 dans le chirurgie des brulés et Luthra [12] 23\% en chirurgie cardiaque.

Dans les pays Anglo-saxons, ce temps d'induction est un élément analysé pour évaluer la performance au bloc opératoire appelé «knife to skin » [12]. Il parait possible d'améliorer ce temps T1 après avoir analysé les différentes étapes entre l'entrée du patient et l'incision pour permettre une meilleure prise en charge [13,14]. Les choix en termes d'anesthésie ont un impact non négligeable sur la fluidité du parcours des patients. Une réflexion peut être menée sur un certains nombres d'aspects :

La mise à disposition de l'espace de pré-induction permet d'anticiper certaines tâches et de réaliser certaines inductions (notamment anesthésies locorégionales) pendant le nettoyage et la préparation de la salle opératoire.

\section{Temps opératoire}

La durée opératoire peut être plus approximative qu'en chirurgie programmée du fait de difficultés opératoires, ou d'erreur de matériel. La venue du malade suivant peut être retardée alors qu'elle est quasi automatique et mieux régulée en chirurgie programmée.

Les temps opératoires sont généralement les plus critiqués par les différents acteurs du bloc opératoire $[12,15]$, hors elles ne représentent ici même pas la moitié de l'occupation réelle de la salle.

Certains auteurs préconisent également des équipes chirurgicales dévouées à la traumatologie pour occuper la salle dédiée afin d'améliorer la prise en charge globale à la fois sur les plans clinique et administratif [16, 17]. Si cette pratique est développée dans les pays Anglo-Saxons, elle reste rare en hexagone [18]. 


\section{Anticiper la préparation du matériel}

Les ressources, notamment matérielles, nécessaires aux interventions doivent être identifiées. Une bonne anticipation de la préparation du matériel permet de contribuer à la fluidité de la réalisation du programme opératoire. L'homogénéisation des pratiques facilite également la préparation du matériel et la gestion des stocks.

\section{Sortie du patient}

Lorsque cela est possible, réveiller les patients en salle de réveil plutôt qu'en salle d'opération permet de libérer la salle plus tôt, permettant de gagner du temps sur l'intervention suivante.

\section{Turn-over}

- Optimiser le bio nettoyage :

Le bio nettoyage entre deux interventions doit être assuré par un personnel identifié selon des protocoles validés par le CLIN, adaptés à chaque spécialité chirurgicale et type d'intervention. Les délais de remise en état des salles entre deux interventions doivent être régulièrement analysés afin d'identifier d'éventuels écarts par rapport au temps moyen prévu. Ces écarts peuvent en effet entraîner des retards dans le programme et des temps d'attente plus longs pour le patient [19].

- Commande du patient suivant :

Le renforcement de la régulation permet d'améliorer l'enchaînement des opérations et d'améliorer le taux d'occupation des salles. Pour faciliter la mission du régulateur, il doit pouvoir suivre l'avancement du programme dans les différentes salles, en temps réel.

- Brancardage : 
L'optimisation du brancardage contribue à améliorer le flux des patients, en particulier aux périodes de pics d'activité, qui se situent généralement soit au démarrage de la journée, soit vers midi. Il peut être opportun de revoir l'affectation des brancardiers selon les pics d'activité de la journée, et les modalités d'appel des brancardiers.

Cette réorganisation du brancardage peut permettre de réduire le délai d'attente des patients à l'entrée et à la sortie.

Favoriser la descente des patients à pied pour les chirurgies programmées et surtout pour le membre supérieur s'avère efficace et permet d'optimiser l'utilisation des brancardiers [20].

Lorsque le malade suivant n'a pas été suffisamment anticipé, cela se répercute sur le temps d'enchaînement entre deux chirurgies. Les gestes normalement réalisés en salle de pré induction (anesthésie locorégionale, mise en place des cathéters) seront alors réalisés en salle d'intervention. Ceci aura pour conséquence d'augmenter le temps d'attente du chirurgien et d'augmenter le TROS sans que le taux d'occupation de salle soit diminué. Ce disfonctionnement sera donc peu visible par les différents indicateurs

L'implication du personnel médical et non médical reste un facteur qui modifie le rendement de l'utilisation de la salle. En effet, contrairement à une salle de chirurgie programmée, il n'y a pas de programme à tenir en salle de traumatologie donc le personnel peut être un peu moins attentif au bon déroulement des enchaînements, d'autant plus que la salle est ouverte $24 \mathrm{~h} / 24$ et que leur temps de travail est posté.

Au final chaque temps d'occupation est allongé en chirurgie d'urgence par rapport à la chirurgie programmée mais sans qu'il y est en terme de ratio de véritable différence en terme de pourcentage d'occupation de salle.

Les principaux axes d'amélioration de l'efficience sont l'arrivée du premier malade en salle de traumatologie et le délai « entrée - incision ».

Le turn-over le week-end doit être optimisé pour optimiser l'utilisation de la salle. 
Afin d'améliorer l'arrivée du premier malade en chirurgie traumatologique. La solution qui nous paraît la plus appropriée est d'utiliser le même fonctionnement que pour la chirurgie orthopédique. La demande de transport pour le bloc opératoire du premier malade serait réalisée la veille sur le logiciel informatique par l'infirmière de garde selon le choix du chirurgien et en accord avec l'anesthésiste.

Si l'ordre devait être modifié, en raison d'une fracture diaphysaire par exemple chez un patient hospitalisé dans la nuit, l'infirmière serait alors capable, sur demande du chirurgien, de modifier cet ordre et de programmer le transport du nouveau patient. L'anesthésiste serait alors prévenu pour réaliser la consultation pré opératoire.

Ainsi l'ensemble des acteurs est informé des caractéristiques concernant le premier malade et a pu vérifier l'absence de manquement (bilan sanguin, matériel, scanner pré opératoire). Le transport pour le bloc opératoire est ainsi défini de manière fiable.

En révélant ces informations et après une formation des équipes, il est possible d'améliorer les pratiques pour permettre une meilleure activité et une meilleure prise en charge des malades que ce soit en chirurgie programmée ou traumatologie [19]. 


\section{CONCLUSION}

L'efficience de la salle de traumatologie était certes moindre par rapport à une salle de chirurgie programmée mais reste somme toute satisfaisant avec un taux d'occupation de salle supérieur à $80 \%$. Chaque étape de l'intervention chirurgicale se trouvait rallongée en traumatologie, mais la proportion de temps d'occupation de salle des différentes étapes était similaire entre les différents groupes. Les principaux axes d'amélioration seraient l'heure d'arrivée du premier patient et le turn-over.

\section{Déclaration d'intérêts}

Aucun des auteurs n'a de conflits d'intérêts à déclarer.

\section{Financement}

Aucun

\section{Contributions individuelles des auteurs}

TR : conception et méthodologie de l'étude ; recueil, analyse, et interprétation des données ; analyse statistique ; rédaction du manuscrit ; approbation de la version définitive soumise AW : conception et méthodologie de l'étude ; recueil, analyse, et interprétation des données JS : approbation de la version définitive soumise

LD : analyse et interprétation des données ; révision critique des éléments intellectuellement importants du manuscrit ; approbation de la version définitive soumise FC : conception et méthodologie de l'étude ; approbation de la version définitive soumise 
Tableau 1 : Moyenne des indicateurs de bloc opératoire

\begin{tabular}{|c|c|c|c|}
\hline & $\begin{array}{l}\text { Groupe A } \\
n=691\end{array}$ & $\begin{array}{c}\text { Groupe B } \\
n=819\end{array}$ & $\begin{array}{l}\text { Groupe C } \\
n=327\end{array}$ \\
\hline TVO & $8 \mathrm{~h} 26 \pm 2 \mathrm{~h} 24$ & $9 \mathrm{~h} 47 \pm 1 \mathrm{~h} 38$ & $10 \mathrm{~h} 55 \pm 4 \mathrm{~h} 09$ \\
\hline TROS & $2 \mathrm{~h} 17 \pm 1 \mathrm{~h} 13$ & $2 \mathrm{~h} 43 \pm 1 \mathrm{~h} 38$ & $2 \mathrm{~h} 11 \pm 1 \mathrm{~h} 03$ \\
\hline TO & $88,4 \% \pm 7 \%$ & $85,6 \% \pm 8 \%$ & $75,7 \% \pm 15 \%$ \\
\hline $\begin{array}{l}\text { Arrivée premier } \\
\text { malade }\end{array}$ & $7 \mathrm{~h} 32 \pm 0 \mathrm{~h} 15$ & $08 \mathrm{~h} 11 \pm 0 \mathrm{~h} 45$ & $09 \mathrm{~h} 05 \pm 0 \mathrm{~h} 49$ \\
\hline $\begin{array}{c}\text { Incision premier } \\
\text { malade }\end{array}$ & $08 \mathrm{~h} 33 \pm 0 \mathrm{~h} 18$ & $09 \mathrm{~h} 16 \pm 0 \mathrm{~h} 48$ & $10 \mathrm{~h} 11 \pm 0 \mathrm{~h} 51$ \\
\hline $\begin{array}{c}\text { T1 : délai entrée - } \\
\text { incision }\end{array}$ & Oh46 \pm 0h19 & $0 \mathrm{~h} 55 \pm 0 \mathrm{~h} 21$ & $0 \mathrm{~h} 39 \pm 2 \mathrm{~h} 03$ \\
\hline $\begin{array}{c}\mathrm{T} 2 \text { : délai incision - } \\
\text { fermeture }\end{array}$ & $01 \mathrm{~h} 05 \pm 0 \mathrm{~h} 55$ & $1 \mathrm{~h} 14 \pm 1 \mathrm{~h} 42$ & $0 \mathrm{~h} 42 \pm 2 \mathrm{~h} 06$ \\
\hline $\begin{array}{c}\text { T3 : délai fermeture - } \\
\text { sortie }\end{array}$ & $0 \mathrm{~h} 27 \pm 0 \mathrm{~h} 44$ & $0 \mathrm{~h} 36 \pm 1 \mathrm{~h} 11$ & Oh31 $\pm 01 \mathrm{~h} 17$ \\
\hline $\begin{array}{c}\text { T4 : Remise en état } \\
\text { de la salle }\end{array}$ & $0 \mathrm{~h} 17 \pm 0 \mathrm{~h} 25$ & $0 \mathrm{~h} 28 \pm 0 \mathrm{~h} 25$ & $0 \mathrm{~h} 58 \pm 1 \mathrm{~h} 08$ \\
\hline T5 : Turn-over & $0 \mathrm{~h} 27 \pm 0 \mathrm{~h} 25$ & $0 \mathrm{~h} 40 \pm 0 \mathrm{~h} 26$ & $1 \mathrm{~h} 11 \pm 1 \mathrm{~h} 16$ \\
\hline
\end{tabular}

TVO : Temps de vacation offert; TROS: Temps réel d'occupation de salle; TO : Taux d'occupation de salle 
Figure 1:

Définition du Temps réel d'occupation de salle (TROS)

Definition of Room Occupancy Time (ROT)

Figure 2 :

Distribution des différents temps d'occupation de salle dans les 3 groupes.

Real time distribution of room occupancy in different groups. 
Références :

[1] Strum DP, Vargas LG, May JH, Bashein G. Surgical suite utilization and capacity planning: a minimal cost analysis model. Journal of medical systems. 1997;21:309-22.

[2] V. Luce AB, C. Girier Diebolt. Intérêt du suivi des indicateurs du bloc opératoire pour l'optimisation des ressources et l'activité chirurgicale. Annales Françaises d Anesthésie et de Réanimation. 2013;32:A214-A215

[3] Lingard L, Espin S, Whyte S, Regehr G, Baker GR, Reznick R, et al. Communication failures in the operating room: an observational classification of recurrent types and effects. Quality \& safety in health care. 2004;13:330-4.

[4] Wolf FA, Way LW, Stewart L. The efficacy of medical team training: improved team performance and decreased operating room delays: a detailed analysis of 4863 cases. Annals of surgery. 2010;252:477-83; discussion 83-5.

[5] Makary MA, Sexton JB, Freischlag JA, Millman EA, Pryor D, Holzmueller C, et al. Patient safety in surgery. Annals of surgery. 2006;243:628-32; discussion 32-5.

[6] Einav Y, Gopher D, Kara I, Ben-Yosef O, Lawn M, Laufer N, et al. Preoperative briefing in the operating room: shared cognition, teamwork, and patient safety. Chest. 2010;137:443-9.

[7] Moss J, Xiao Y. Improving operating room coordination: communication pattern assessment. The Journal of nursing administration. 2004;34:93-100.

[8] Sasano N, Morita M, Sugiura T, Sasano H, Tsuda T, Katsuya H. Time progression from the patient's operating room entrance to incision: factors affecting anesthetic setup and surgical preparation times. Journal of anesthesia. 2009;23:230-4. 
[9] Thomasson Ed, Bonfait H, Delaunay C, Charrois O, Orthorisq. Patient safety in the operating room: Is the "checklist" implementation sufficient to improve safety? A survey at 6 months follow-up of routine enforcement. Revue de chirurgie orthopédique et traumatologique. 2011;97:869-76.

[10] Haynes AB, Weiser TG, Berry WR, Lipsitz SR, Breizat AH, Dellinger EP, et al. A surgical safety checklist to reduce morbidity and mortality in a global population. The New England journal of medicine. 2009;360:491-9.

[11] Madni TD, Imran JB, Clark A, Arnoldo BA, Phelan HA, 3rd, Wolf SE. Analysis of operating room efficiency in a burn center. Journal of burn care \& research : official publication of the American Burn Association. 2017.

[12] Luthra S, Ramady O, Monge M, Fitzsimons MG, Kaleta TR, Sundt TM. "Knife to skin" time is a poor marker of operating room utilization and efficiency in cardiac surgery. Journal of cardiac surgery. 2015;30:477-87.

[13] Mariano ER, Lehr MK, Loland VJ, Bishop ML. Choice of loco-regional anesthetic technique affects operating room efficiency for carpal tunnel release. Journal of anesthesia. 2013;27:611-4.

[14] Richman JM, Stearns JD, Rowlingson AJ, Wu CL, McFarland EG. The introduction of a regional anesthesia rotation: effect on resident education and operating room efficiency. Journal of clinical anesthesia. 2006;18:240-1.

[15] Merat S, Tortosa JC, Vincenti-Rouquette I, Fevre G, Rousseau JM. [Organization of an operational site. Comparison of the durations of intervention planned and real]. Ann Fr Anesth Reanim. 2006;25:152-7. 
[16] Brusalis CM, Shah AS, Luan X, Lutts MK, Sankar WN. A dedicated orthopaedic trauma operating room improves efficiency at a pediatric center. The Journal of bone and joint surgery American volume. 2017;99:42-7.

[17] Heng M, Wright JG. Dedicated operating room for emergency surgery improves access and efficiency. Canadian journal of surgery Journal canadien de chirurgie. 2013;56:167-74.

[18] Althausen PL, Kauk JR, Shannon S, Lu M, O'Mara TJ, Bray TJ. Operating room efficiency: benefits of an orthopaedic traumatologist at a level II trauma center. Journal of orthopaedic trauma. 2014;28:e101-6.

[19] Champault A, Arsena V, Barrat C, Bayeh P, Champault G. Can the time between interventions be reduced in the operating theater? Prospective study. Annales de chirurgie. 2003;128:599-602.

[20] J.Guezennec GK, C.Menigaux, L.Boudon, M.Chauvin. Optimisation du flux des patients dans un bloc d'orthopédie. réorganisation des modalités d'accueil et circulation a pied des patients. Annales Françaises d'Anesthésie et de Réanimation. 2014; Volume 33, Supplement 2:Page A344. 


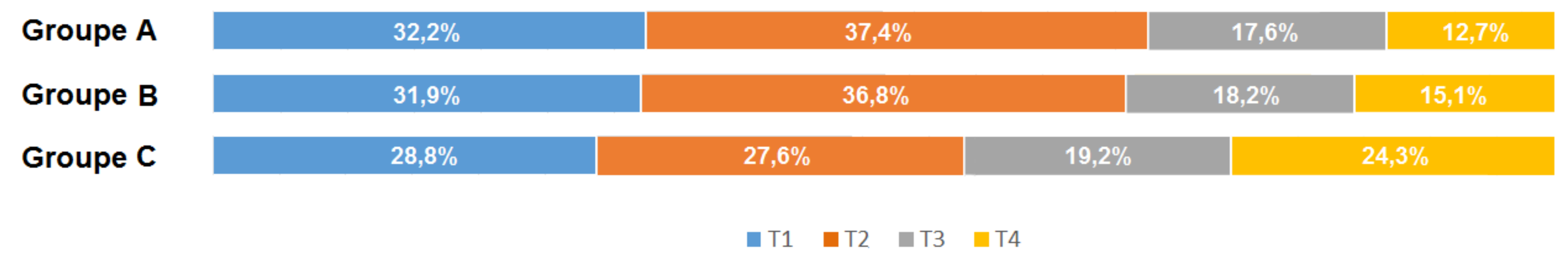

